\title{
Dislocation-Free GaN Nanowires
}

\author{
J.P. Zhang ${ }^{*}$, Y. Wu, G. S. Cheng ${ }^{* *}$, M. Moskovits ${ }^{* *}$, J.S. Speck ${ }^{*}$ \\ *Materials Department, University of California, Santa Barbara, CA 93106 \\ **Chemistry Department, University of California, Santa Barbara, CA 93106
}

In this work we report on the structural characteristics of dislocation-free wurzite GaN nanowires. The GaN nanowires were produced by a controllable vapor-liquid-solid process in the presence of an indium catalyst [1] and can be doped either n-type or p-type. Commonly, the nanowires either exhibit the GaN crystallographic c-axis $(\sim 15-20 \%$ of the fibers) or $\langle 1 \overline{1} 00\rangle$ direction (m-direction, $\sim 70 \%$ of the fibers) parallel to the fiber axis. Other fibers exhibit higher index growth directions.

The nanowires exhibit lengths as great as $15 \mu \mathrm{m}$ and diameters in the range $10-200 \mathrm{~nm}$. Two parameters are found to be crucial to the microstructure of $\mathrm{GaN}$ nanowires: $(i)$ the growth axis; and (ii) the nanowire diameter. We have found that c-axis oriented nanowires are highly defective. Similarly, m-direction nanowires with diameters less than $\sim 20 \mathrm{~nm}$ are also defective (the structure of defective papers will be addressed in a separate paper). However, m-direction fibers with diameters in the range $20 \mathrm{~nm}-400 \mathrm{~nm}$ are free from extended defects. In this paper, we concentrate on the structure of the m-direction fibers.

Scattering contrast studies in two beam conditions (typically recorded either with $\mathbf{g}=1 \overline{2} 10$ or $\mathrm{g}=$ $1 \overline{1} 00$ ) from a number of m-axis nanowires verify the absence of extended defects. Typical images are shown in Fig. 1. As known from studies of epitaxial GaN thin film, $\mathbf{g}=1 \overline{2} 10$ and $\mathrm{g}=1 \overline{1} 00$ two beam imaging conditions can be used to identify all common planar defect and threading dislocations (except for those with a pure c-axis Burgers vector) in hexagonal GaN. The scattering contrast images shown in Fig. 1 have a large variation in the excitation error along the fiber axis due to both bending and twisting of the fiber.

High resolution images (JEOL 2010, $1.9 \AA$ resolution) of the fibers recorded down the [0001] and $[1 \overline{2} 10]$ zone axis reveal their local surface structure, as shown in Fig. $2 \mathrm{a}$ and $2 \mathrm{~b}$, respectively. The surfaces of the nanowires are clean (no evidence of a native oxide or amorphous phases) and appear to be free from contamination even after air exposure for six months (EELS studies have shown no evidence for a carbonaceous layer on the fibers). The [0001] zone axis high resolution images show the surface is comprised of $\{1 \overline{1} 00\}$ faces. Viewing down the [1 $\overline{2} 10]$ zone axis shows that the fibers also have clean (0001) stepped facets. The schematic structure is shown in Fig. 2c. Finally, the high resolution images verify the absence of stacking faults.

\section{References}

[1] Guosheng Cheng et al., Appl. Phys. Lett., (to be published)

[2] This research was supported by Department of Energy under contract number 8-482490-25005.

The work also made use of the MRL Center Facilities at University of California, at Santa Barbara. 


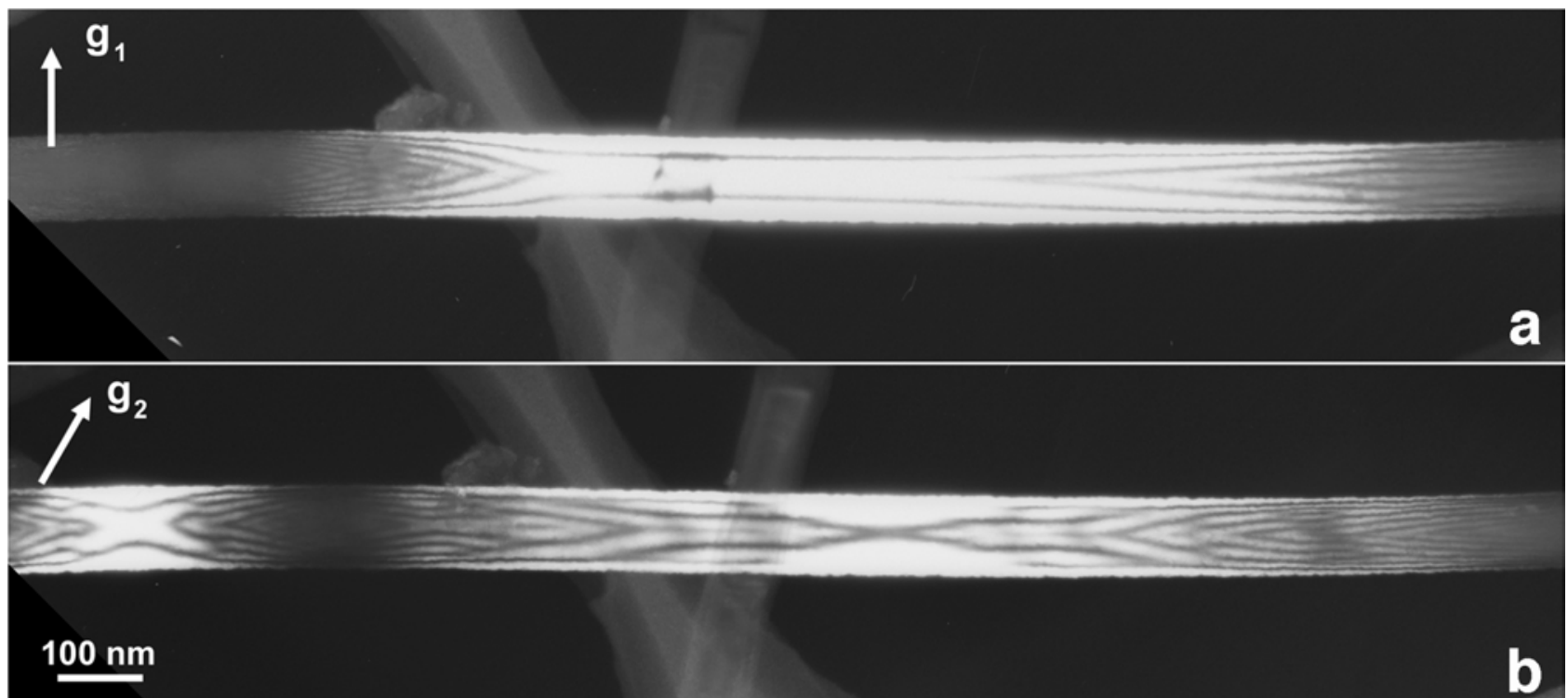

Figure 1. Dislocation-free $\mathrm{GaN}$ nanowires have been identified with $(\mathbf{0}, \mathbf{g})$ imaging, where $\mathrm{g}=1 \overline{2} 10$ in (a) and $\mathbf{g}=1 \overline{1} 00$ in (b). The changes in spacing of the thickness fringes is due to the rocking of the $\mathrm{c}$ axis located.
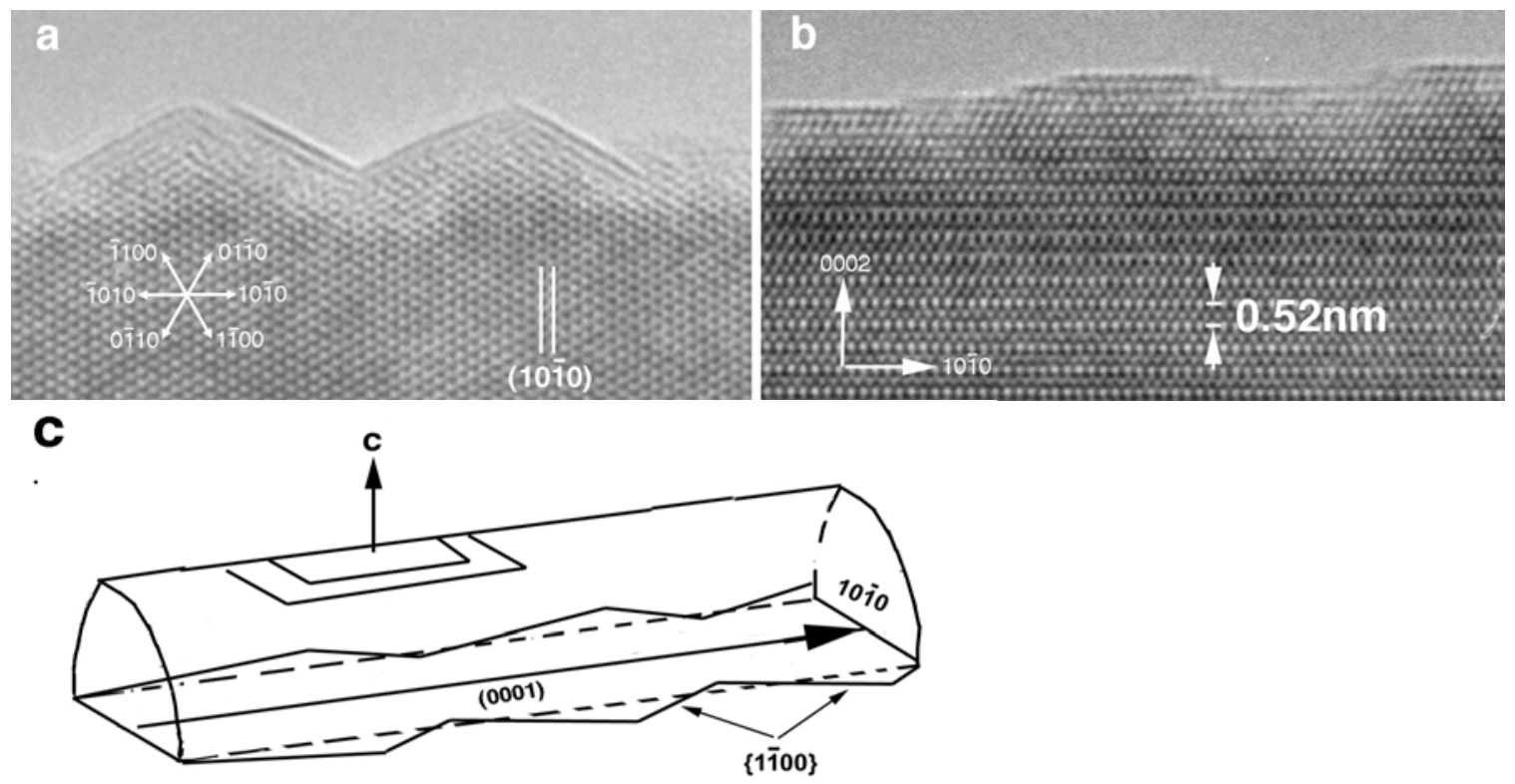

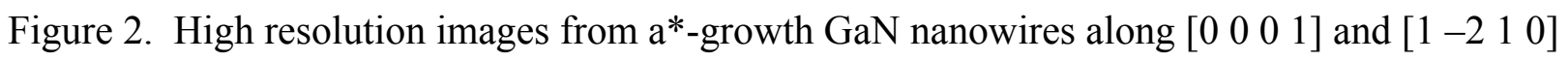
indicate that the nanowires are not only dislocation-free, but also having clean surfaces that the $\{-1$ $100\}$ facets in (a) and the c-plane surface steps in (b) are clear to see. The schematic fiber structure is shown in part (c). 